\title{
On the physics of fizzing: How bubble bursting controls droplets ejection
}

\author{
Elisabeth Ghabache, Arnaud Antkowiak, Christophe Josserand, Thomas Séon ${ }^{1}$ \\ Sorbonne Universités, Université Pierre et Marie Curie and Centre National de la Recherche Scientifique, \\ Unité Mixte de Recherche 7190, Institut Jean Le Rond d'Alembert, 4 Place Jussieu, F-75005 Paris, \\ France
}

(Dated: 29 October 2018)

\begin{abstract}
Bubbles at a free surface surface usually burst in ejecting myriads of droplets. Focusing on the bubble bursting jet, prelude for these aerosols, we propose a simple scaling for the jet velocity and we unravel experimentally the intricate roles of bubble shape, capillary waves, gravity and liquid properties. We demonstrate that droplets ejection unexpectedly changes with liquid properties. In particular, using damping action of viscosity, self-similar collapse can be sheltered from capillary ripples and continue closer to the singular limit, therefore producing faster and smaller droplets. These results pave the road to the control of the bursting bubble aerosols.
\end{abstract}

Savoring a glass of champagne would not be as enjoyable without this fizzy sensation coming from bursting bubbles at the surface. More than just triggering a simple tingling sensation, the tiny droplets ejected during bursting are crucial for champagne tasting as their evaporation highly contribute to the diffusion of wine aroma in air 1 . Airborne droplets resulting from sea surface bubble bursting are also known since the late forties $^{2} 4$ to play a major role in the interaction between ocean and atmosphere 516 . Two distinct types of droplets are involved, lying on two different mechanisms appearing during bubble bursting 7 . When the thin liquid film - the bubble cap - separating the bubble from the atmosphere disintegrates, film drops are produced ${ }^{\sqrt[8]{9}}$ with radius mainly less than $1 \mu \mathrm{m}$. Then the resulting opened cavity (see Fig 1 collapses and a jet emerges producing jet drops by breaking up 10 . For example, this latter mechanism accounts for the majority of sea-spray aerosol particles in the atmosphere with radius between 1 and $25 \mu \mathrm{m} 13$.

The last sixty years have witnessed a number of laboratory studies documenting jet drops properties, such as the ejection speed, the maximum height or the size distribution as a function of bubble volume ${ }^{7 / 14}[16 \mid 19$, but a comprehensive picture of the mechanisms at play in bubble bursting is still lacking. In particular, the sequence of violent events preluding jet formation $\frac{11 \mid 17}{17}$ and the roles of liquid properties remain elusive.

In this article, we unravel the tangled roles of liquid properties, gravity and capillary waves in the cavity collapse and show that these waves invariably adopt a self-similar behavior. We evidence the critical role of viscosity, that shelters self-similar collapse from remnant ripples, and therefore promotes the emergence of thinner and faster jets. Optimal conditions for singular jets as well as general scaling laws for the jet dynamics are assessed from detailed bubble bursting experiments. The consequences for aerosol generation are finally outlined, in particular in the context of champagne fizz, where liquid properties are tunable.

Our experiment consists in releasing a gas bubble from a submerged needle in a liquid and recording the upward jet after the bubble bursts at the free surface. Bubbles are quasi-steadily formed using a syringe pump and detachment frequency is weak enough to avoid successive bubbles interaction. Different needle diameters $(5<\Phi(\mu \mathrm{m})<1800)$ allow us to create bubbles with various radii $(R)$ ranging from $300 \mu \mathrm{m}$ to $2000 \mu \mathrm{m}$. The liquids used in this study include nine water-glycerol mixtures of viscosity in the range $\mu=1$ mPa.s - $12 \mathrm{mPa} . \mathrm{s}$, surface tension $\gamma=64 \mathrm{mN} \cdot \mathrm{m}^{-1}-72 \mathrm{mN} . \mathrm{m}^{-1}$, and density $\rho=1000 \mathrm{~kg} . \mathrm{m}^{-3}$ $1160 \mathrm{~kg} \cdot \mathrm{m}^{-3}$ and ethanol $\left(\mu=1.2 \mathrm{mPa} . \mathrm{s}, \gamma=23 \mathrm{mN} \cdot \mathrm{m}^{-1}, \rho=780 \mathrm{~kg} . \mathrm{m}^{-3}\right)$. The height of fluid between needles and free surface is kept short $(2-3 \mathrm{~cm})$ to avoid rising bubble inflation. The bubble collapse and jet dynamics are analyzed through extreme close-up ultra-fast imagery. Macro lenses and extension rings allow us to record with a definition reaching $5 \mu \mathrm{m}$ per pixel. Images are obtained between 10000 and 150000 frames per second using a digital high-speed camera (Photron SA-5). The ejection speed $V_{\text {tip }}$ is measured when the tip of the jet reaches the mean water level.

Figure 1 (see also Videos S1 and S2) illustrates a typical jetting event following a bubble bursting at a free surface in water. The top sequence shows the free surface view while the bottom one displays the underwater dynamics. The first image of the top sequence shows a static bubble lying at the free surface. Then the film separating the bubble from the atmosphere drains and bursts leaving an unstable opened cavity. This cavity is millimeter-sized so the restoring force which tends to bring this hole back to a flat equilibrium is capillary driven. Bottom sequence displays capillary waves propagating along this cavity and focusing at the bottom. These collapsing waves give rise to a high speed vertical jet shooting out above the free surface as observed on the top sequence. The jet then fragments into droplets due to Rayleigh-Plateau destabilization generating an aerosol of one to ten droplets 7 . 


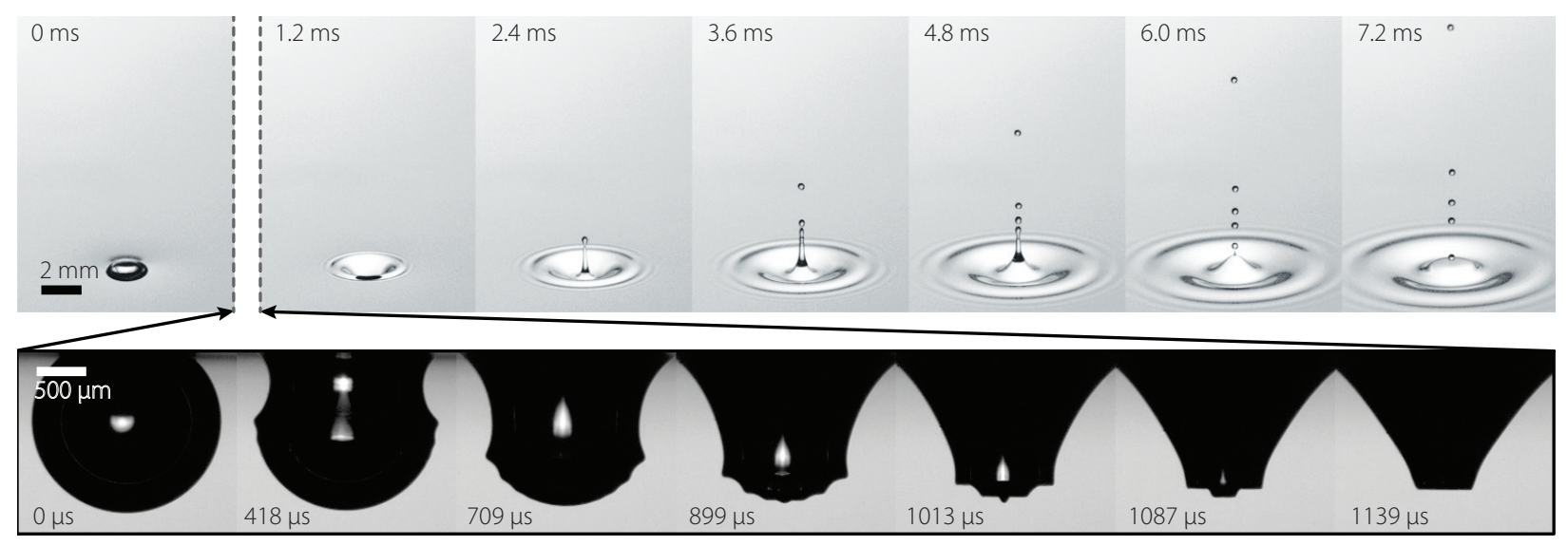

FIG. 1. Time sequence of a typical jetting event following a bubble bursting at a free surface in water. The top sequence shows the bubble bursting event above the free surface, while the bottom sequence displays the bubble under the free surface during the collapse giving birth to the vertical upward jet. The bottom sequence takes place between the two first images of the top one. The times are shown on the snapshots with the same origin. See also the corresponding Videos S1 and S2.

In order to establish the role played by the relevant parameters in the jet dynamics, we identify the five variables ruling the value of the jet tip velocity:

$$
V_{\mathrm{tip}}=\Phi(R, \rho, \gamma, g, \mu)
$$

Using dimensional arguments, this equation becomes a relation between three dimensionless numbers fully describing the jet dynamics:

$$
\mathrm{We}=F(\mathrm{Bo}, \mathrm{Mo})
$$

where the Weber number We $=\rho V_{\text {tip }}^{2} R / \gamma$ compares the effect of inertia and capillarity on the jet dynamics, the Bond number Bo $=\rho g R^{2} / \gamma$ compares the effect of gravity and capillarity on the initial bubble and the Morton number Mo $=g \mu^{4} / \rho \gamma^{3}$ only depends on the fluid properties and is in particular independent of the bubble radius $R$. Various scaling relations for the velocity are reported in the literature, ranging from exponential fits of experimental data ${ }^{\sqrt{19}}$ (see curved dashed line Fig. 22 to algebraic laws $V_{\text {tip }} \propto R^{-1 / 2}$ in numerical simulations disregarding gravity 17 . This diversity certainly emphasizes the need for further experimental analysis.

We set out by investigating experimentally in Fig. 2 the dependence of $V_{\text {tip }}$ with $R$ in a log-log plot. Our experimental data (circles) rest along the line $V_{\text {tip }} \propto R^{-1}$, as indicated by the red dashed line fitting the experimental velocities. Note that bubbles with Bo $>1$ (radii greater than $3 \mathrm{~mm}$ ) are out of scope of this study because they give rise to jets with a different dynamics and would constitute an other study. On the same figure various data from the literature have been plotted: the top drop velocity measured experimentally in fresh water ${ }^{[19}$ or in sea water ${ }^{7}$, and the maximum tip velocity of the jet computed numerically in fresh water 16 . It is noteworthy that our jet velocities match the first drop velocities (fitted by the exponential dashed line) making our results relevant for aerosol generation. Regardless of some slight differences they all follow the same trend $V_{\text {tip }} \propto R^{-1}$. This specifies the form of the Eq. 11 providing the variation with Bond number, yielding:

$$
\mathrm{We}=\mathrm{Bo}^{-1 / 2} f(\mathrm{Mo})
$$

The $R^{-1}$ behavior is the footprint of gravity effects: the introduction of a second length scale, the gravitycapillary length $\ell_{\mathrm{gc}}=\sqrt{\gamma / \rho g}$, allows departures from capillaro-inertial predictions through length scales ratios 20 : We $=\left(\ell_{\mathrm{gc}} / R\right) f(\mathrm{Mo})$. Though the Bond number remains small in the experiments, the gravity plays a genuine role in the collapse dynamics that needs to be elucidated. Froude number of the jet at the mean water level is $\mathrm{Fr}=V_{\text {tip }} / \sqrt{g R}=\sqrt{\mathrm{We} / \mathrm{Bo}}$, and can be expressed here as $\mathrm{Fr}=\mathrm{Bo}^{-3 / 4} f(\mathrm{Mo})^{1 / 2}$. In water, with the Bond values of Fig 2 one obtains Fr $\in[7-160]>1$, indicating that gravity hardly affects the jet dynamics at least before eruption. 


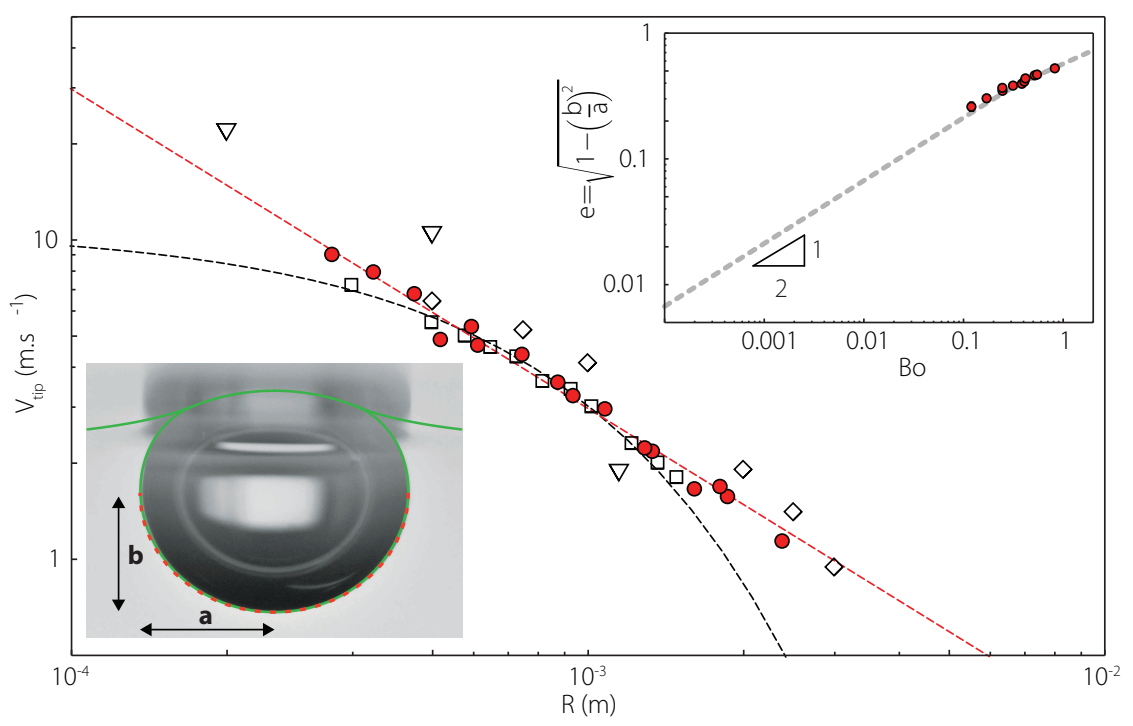

FIG. 2. Jet velocity $V_{\text {tip }}$ as a function of the bubble radius $R$ in water from our experiments (red circle) and data from the literature: (square) top drop velocity from Spiel et al ${ }^{[19}$ along with their exponential fit represented by the curved dashed line $V=10.72 \mathrm{e}^{-1.27 .10^{3} R}$ measured experimentally in fresh water, (diamonds) maximum tip velocity computed numerically in fresh water from Boulton-Stone et al ${ }^{[16}$ (triangle) top drop velocity from Blanchard ${ }^{17}$ The red dashed line is a fit of our experimental data exhibiting the common trend $V_{\text {tip }}=\xi R^{-1}\left(\xi=2.9510^{-3} \mathrm{~m}^{2} . \mathrm{s}^{-1}\right)$. Bottom left inset: picture of a static floating bubble at the free surface. Green profile is obtained by numerical integration of the YoungLaplace equation using MATHEMATICA software ${ }^{9 \mid 18}$. The red dashed line is a semi-elliptic fit of the bottom part of the static bubble. $a$ and $b$ identify respectively the semi-minor and semi-major axis of the ellipsoid. Top right inset: static bubble eccentricity $e=\sqrt{1-(a / b)^{2}}$ computed numerically as a function of the Bond number (dashed line). The red circles correspond to our experiments in water. Dashed line show asymptotic behaviors $e \propto \mathrm{Bo}^{1 / 2}$ for small Bond number.

Bottom left inset of Fig. 2 displays a picture of the static bubble before bursting. Superimposed on the experimental picture, the profile is obtained by numerical integration of the YoungLaplace equation using Mathematic $A^{9[18}$. The dashed line is a semi-elliptic fit of the bottom part of the static bubble allowing us to define the bubble parameters: $a, b$ and the corresponding bubble radius $R=\left(a^{2} b\right)^{1 / 3}$. On the top right inset of Fig 2 the eccentricity of the static floating bubble $e$ computed numerically is plotted versus the Bond number. $e$ is found to decrease with the Bond number following $e \propto \mathrm{Bo}^{1 / 2}$, demonstrating the non-sphericity of even small bubbles. This result naturally suggests that gravity influences the jet dynamics not through its direct action on the dynamics but by affecting the initial bubble shape.

We now investigate how the jet eruption velocity $V_{\text {tip }}$ depends on the liquid properties and therefore on the Morton number. The Weber number is plotted as a function of the Bond number for various Morton number on Fig. 3(a). To browse the Morton range we mainly change the liquid viscosity. Correspondence between Morton number, liquid viscosity and symbols is indicated in the table of Fig. 3. The first clear observation is that the jet dynamics depends on the viscosity although the jet Reynolds number is greater than 1. Furthermore, the regime $\mathrm{We} \propto \mathrm{Bo}^{-1 / 2}$ is retained on around four decades in Morton number, from $1 \mathrm{mPa} . \mathrm{s}$ to around $7.5 \mathrm{mPa} . \mathrm{s}$, all plotted with filled markers. This defines the boundary of our study considering that this viscous regime characterized by $\mu \gtrsim 9 \mathrm{mPa} . \mathrm{s}$ and showed with empty markers is out of the scope of this paper. Finally, for values of viscosity less than $6 \mathrm{mPa}$.s we observe a surprising increase of the Weber number with Morton number, meaning that for a given bubble radius in this range, the jet is drastically faster as the liquid viscosity is increased.

The non-dimensional jet velocity $\mathrm{We} \mathrm{Bo}^{1 / 2}$ is plotted as a function of the Morton number on Fig. 3(b), therefore specifying $f(\mathrm{Mo})$ (see Eq. 2). A bell shaped curve is clearly observed with a maximum for $\mu=5.2$ $\mathrm{mPa} . \mathrm{s}$. To illustrate this unexpected behavior we display inside Fig. 3 four snapshots of the jet at the same dimensionless time $\left(\mathrm{t} / \sqrt{\rho R^{3} / \gamma}=1 / 5\right)$, same Bond number $(\mathrm{Bo} \simeq 0.14)$ but four different Morton numbers. The Videos M1, M2 and M3 correspond to the snapshots (1), (2) and (3). The jet morphology undergoes a neat qualitative change as the liquid gets more viscous: the jet first becomes thinner, detaching more and smaller droplets and then ends up fat and small for high Morton number. 


\begin{tabular}{|c|c|c|c|c|c|c|c|c|c|c|}
\hline & $\circ$ & 口 & $\triangleleft$ & 0 & $\Delta$ & $\diamond$ & $\Rightarrow$ & $\triangleright$ & 0 & $\Delta$ \\
\hline$\mu(\mathrm{mPa} . \mathrm{s})$ & 1 & 1.2 Eth & 2.1 & 3.3 & 4.4 & 5.2 & 6.2 & 7.4 & 9.7 & 12 \\
\hline Mo & $2.610^{-11}$ & $2.110^{-9}$ & $6.010^{-10}$ & $3.910^{-9}$ & $1.110^{-8}$ & $2.310^{-8}$ & $4.910^{-8}$ & $9.810^{-8}$ & $2.910^{-7}$ & $6.710^{-7}$ \\
\hline
\end{tabular}
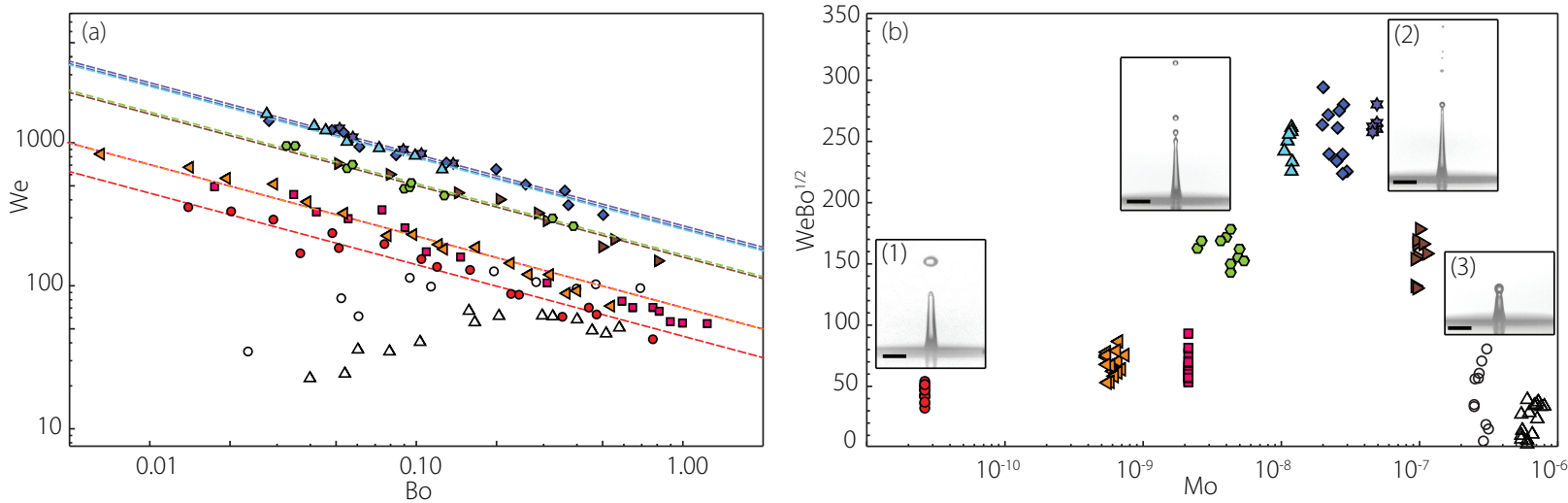

FIG. 3. Table: Value of the liquid viscosity and of the associated Morton number corresponding to each symbol. Eth. stands for Ethanol . (a) Weber number as a function of the Bond number for various values of the Morton number. All the colored symbols follow the same trend $\mathrm{We} \propto \mathrm{Bo}^{-1 / 2}$ as showed by the dashed lines. (b) We Bo ${ }^{1 / 2}$ as a function of the Morton number Mo. Four snapshots display the typical jet observed at the same dimensionless time $\left(\mathrm{t} / \sqrt{\rho R^{3} / \gamma}=1 / 5\right)$, same Bond number $(\mathrm{Bo} \simeq 0.14)$ and four different Morton numbers corresponding to red circle, green hexagon, purple star, empty triangle. See also the Videos M1, M2 and M3 corresponding to the snapshots (1), (2) and (3). The black bar represents $500 \mu \mathrm{m}$

In order to grasp the mechanisms leading to such a particular dynamics, we now turn to the jet formation by focusing on the cavity collapse per se. Lower sequence of Fig. 1 and Video S2 display a typical bubble collapse in water, where a train of capillary waves propagates, converges to the nadir (bottom of the cavity), and gives rise to the jet. Fig 4 shows a temporal zoom of the last microseconds before the cavity collapses $\left(t \simeq t_{0}\right)$ for three different Morton numbers and same Bond number. These three sequences (a), (b) and (c) are the cavity collapse leading to the three jets (1), (2) and (3) displayed on Fig. 3 On the last image of each sequence the cavity is reversed and the upward jet (not seen on the picture) is developing. These sequences show that the cavity reversals are very similar between the 6.2 and $12 \mathrm{mPa}$.s solutions and drastically different from water. In particular the small capillary waves present in the water collapse (a) have disappeared for higher viscosities (b) and (c). It has been shown in numerical simulation ${ }^{17}$ and in other experimental contexts ${ }^{21 \mid 22}$ that such collapse exhibits a self-similar dynamics that can lead in some cases to very thin and rapid jets. In such a situation, the cavity collapses through a nonlinear balance between capillary force and inertia, leading to a self-similar behavior where the lengths scale like $\left(\gamma\left(t_{0}-t\right)^{2} / \rho\right)^{1 / 3} \underline{23}$ ( $t_{0}$ corresponding to the instant of the singular collapse). In the three cases presented here, the same self-similar collapse is clearly at play, as shown on Figs 4(d,e) where the different cavity profiles plotted at different times before $t_{0}$ collapse when lengths are divided by $\left(t_{0}-t\right)^{2 / 3}$. So we observe a capillary-inertia self-similar collapse for each case, even though the jets show clear differences (see Fig. 3 (1), (2) and (3)) and are not singular. Interestingly, the self-similar collapses for the high viscosity cases (b) and (c) are identical, consequently increasing the viscosity leads naturally to slow down the jet. However, these collapses are strongly different from the collapse in water (a) which is perturbed by the presence of the small capillary waves. These waves are always traveling on top of the interface and are inherent to the complex dynamics. But we observe that increasing the viscosity leads to smoothing the collapse.In particular the closest time to $t_{0}$ in water, represented by the dashed profile, do not coalesce properly, signifying that the dynamics is no more self-similar. This results in a collapse leaving its self-similar regime sooner than in a more viscous case, when the remnant ripples are damped. By defining $\mathrm{L}_{\min }$ as the width of the small left cavity when the collapse just quitted it self-similar behavior, Fig. 4(f) shows the variation of this effective collapsing cavity giving rise to the jet and reveals that it decreases with Morton number. This agrees with the idea of a self-similar collapse getting closer to the singularity as viscosity is increased and justifies why the jet velocity is increasing with Morton number.

This suggests an original mechanism to explain the role of the capillary waves for small Morton numbers. We consider that these waves break the self-similar dynamics when they are large enough (typically, when the 


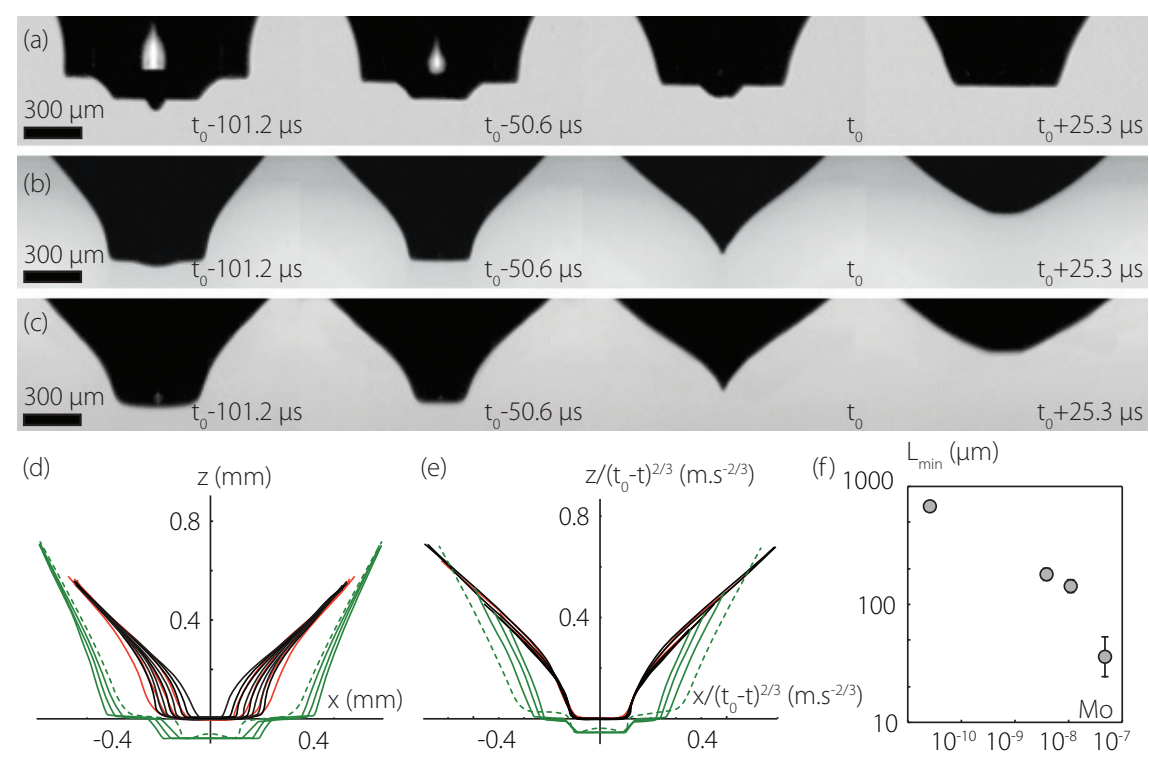

FIG. 4. Snapshots of the final stage of the cavity collapse before the jet develops. (a), (b) and (c) correspond respectively to the jets (1), (2) et (3) of Fig. 3 , $t_{0}$ identifies the time of the wave collapse giving rise to the jet. The cavity profiles corresponding to the three sequences are plotted at different times on (d) (green for sequence (a), black for (b) and red for (c)). (e) shows the collapse of these profiles according to the capillary-inertia self-similar behavior where lengths scale with $\left(t_{0}-t\right)^{2 / 3}$. (f) $\mathrm{L}_{\min }$ versus Morton number. $\mathrm{L}_{\text {min }}$ defines the width of the small left cavity when the collapse just quitted it self-similar behavior. The size of this effective cavity giving rise to the jet eruption decreases with viscosity for $\mu \lesssim 6 \mathrm{mPa}$.s.

wave amplitude is of the order of the self-similar structure). Because the phase velocity of the capillary waves yields $c \propto \sqrt{\gamma k / \rho}$, the shorter the wave, the faster it converges to the nadir, suggesting that the singular dynamics is destroyed by small waves first. This picture has now to be corrected by the viscous damping of the capillary waves ${ }^{24}$ which is also increasing with the wave number (with damping rate $\propto \mu k^{2} / \rho$ ). Therefore, as the viscosity increases, the interface is smoothed near the nadir and the instant where the oscillations destroy the self-similar dynamics is delayed closer and closer to the singularity.

Finally, two regimes of the jet dynamics as a function of the Morton number have been pointed out: for Mo $\lesssim 3.10^{-8}$ the viscosity promotes the jet velocity by smoothing the collapsing cavity and for Mo $\gtrsim 3.10^{-8}$ the jet velocity decreases with viscosity. At the frontier of these two regimes the jet is very thin and its velocity is maximal, which defines a region of the space phase where the aerosol production from bursting bubble is strongly enhanced.

The results presented in this paper apply for bubbles in newtonian fluids with liquid properties such that Bo $\in\left[10^{-2}-1\right]$ and $\mathrm{Mo} \in\left[10^{-11}-10^{-7}\right]$, which include most of the existing bursting bubble aerosols. For instance, Bond and Morton numbers of champagne, from serving temperatures $4{ }^{\circ} \mathrm{C}$ to room temperature, lie usually in the range $\left[8.10^{-3}-8.10^{-1}\right]\left[6.10^{-10}-7.10^{-9}\right]$ respectively, where the droplets ejection sharply depends on the liquid properties (see Fig 3). These results are thus crucial in the context of champagne industry. Indeed, quite recently ${ }^{1}$, ultrahigh resolution mass spectrometry was used in order to analyze the aerosols released by bursting bubbles in champagne. In comparison with the bulk, champagne droplets were found to be over-concentrated with various surface active compounds, some of them showing indeed aromatic properties. This very characteristic fizz is therefore strongly believed to enhance the flavor sensation above a glass of bubbly wine in comparison with that above a glass of flat wine. It is now also well-known that specific treatment on champagne glass enables to create monodisperse bubbles reaching the surface at a chosen radius ${ }^{25 \mid 26}$ and carboxymethyl cellulose (E466), used in food science as a thickener, enables to modify the champagne viscosity with no consequences on the taste ${ }^{27}$. Therefore our results, by evidencing the existence of an unexpected maximum in the aerosols ejection speed and by providing this function between the first drop velocity and the bubble radius and liquid properties, pave the road to the characterization and control of the bursting bubble aerosols. They then constitute an important step forward to the fine tuning of champagne aroma diffusion, major goal of this industry. As an example, after determining the variation of the first droplet radius $r_{d}$ with Bond and Morton number and because we know its velocity, the vertical 
extension of the aerosols could easily be tuned. This will constitute a key result in the control of gaseous exchange between the aerosol and its surroundings. On the other hand, in the context of marine aerosols the Morton number ranges approximatively from $10^{-11}$ to $10^{-9}$, which is in the flat region of the jet velocity dependance on Mo, meaning that hydrodynamical properties of ocean, notably changing with temperature, barely affects the sea spray production. In this context quantity of results have been obtained characterizing the aerosols from bubble in water ${ }^{13}$. However, complementary studies on the number of droplets or drop size distribution as a function of bubble radius and liquid properties, based on the understanding of phenomena described here, will need to be realized to entirely characterize bursting bubble aerosols.

The Direction Générale de l'Armement (DGA) is acknowledged for its financial support. We thank Gérard Liger-Belair for stimulating discussions about the relevance of our results in the champagne context, and for pointing out carboxymethyl cellulose as a possible viscosity modifier for champagne. We also thank Lucas Joseph for running the preliminary experiments.

${ }^{1}$ Liger-Belair G., Cilindre R., Gougeon R. D., Lucio I., Jeandet P., Schmitt-Kopplin P., (2009) Unraveling different chemical fingerprints between a champagne wine and its aerosols. Proc. Natl. Acad. Sci. USA 106:1654516549

${ }^{2}$ Woodcock A. H., Gifford M. M., (1949) Sampling atmospheric sea-salt nuclei over the ocean. Journal of Marine Research 8:177

${ }^{3}$ Woodcock A. H., (1952) Atmospheric salt particles and raindrops. Journal of Meteorology 9:200

${ }^{4}$ Woodcock A. H., Kientzler C. F., Arons A. B., Blanchard D. C., (1953) Giant Condensation Nuclei from Bursting Bubbles. Nature 172:1144

${ }^{5}$ Andreas E. L., Edson J. B., Monahan E. C., Rouault M. P., Smith S. D., (1995) The spray contribution to net evaporation from the sea: A review of recent progress. Boundary-Layer Meteorol. $\mathbf{7 2}$

${ }^{6}$ de Leeuw G., Andreas E. L., Anguelova M. D., Fairall C. W., Lewis E. R., ODowd C., Schulz M., Schwartz S. E., (2011) Production flux of sea spray aerosol. Rev. Geophys. 49

${ }^{7}$ Blanchard D. C., (1963) The electrification of the atmosphere by particles from bubbles in the sea. Progress In Oceanography 1:73

${ }^{8}$ Knelman F., Dombrowski N., Newitt D. M., (1954) Mechanism of the bursting of bubbles. Nature 173:261

${ }^{9}$ Lhuissier H., Villermaux E. (2012) Bursting bubble aerosols. Journal of Fluid Mechanics 696:5-44

${ }^{10}$ Stuhlman Otto, (1932) The mechanics of effervescence. Physics 2:457-466

${ }^{11}$ MacIntyre F. (1972) Flow patterns in breaking bubbles. Journal of Geophysical Research 77:5211-5228

${ }^{12}$ Spiel D.E., (1997) More on the births of jet drops from bubbles bursting on seawater surfaces. J. Geophys. Res. 102:5815-5821

${ }^{13}$ Lewis E. R., Schwartz S. E., (2004) Sea Salt Aerosol Production. Mechanisms, Methods, Measurements, and Models. Ed. Geophysical Monograph 152. (American Geophysical Union, Washington, DC)

${ }^{14}$ Hayami S., Toba Y., (1958) Drop Production by Bursting of Air Bubbles on the Sea Surface (I) Experiments at Still Sea Water Surface. Journal of the Oceanographical Society of Japan 14:145-150

${ }^{15}$ Wu J., (1973) Spray in the atmospheric surface layer: Laboratory study. Journal of Geophysical Research 78:511-519

${ }^{16}$ Boulton-Stone J. M., Blake J. R., (1993) Gas bubbles bursting at a free surface. Journal of Fluid Mechanics 254:437-466

${ }^{17}$ Duchemin L., Popinet S., Josserand C., Zaleski S., (2002) Jet formation in bubbles bursting at a free surface. Phys. Fluids 14:3000-3008

${ }^{18}$ Toba Y., (1959) Drop Production by Bursting of Air Bubbles on the Sea Surface (II) Theoretical Study on the Shape of Floating Bubbles. Journal of the Oceanographical Society of Japan 15:121-130

${ }^{19}$ Spiel D.E., (1995) On the births of jet drops from bubbles bursting on water surfaces. J. Geophys. Res. 100:4995-5006

${ }^{20}$ Seon T., Antkowiak A., (2012) Large bubble rupture sparks fast liquid jet. Phys. Rev. Lett. 109:014501

${ }^{21}$ Zeff B. W., Kleber B., Fineberg J., Lathrop D. P., (2000) Singularity dynamics in curvature collapse and jet eruption on a fluid surface. Nature 403:401-404

${ }^{22}$ Bartolo D., Josserand C., Bonn D., (2006) Singular Jets and Bubbles in Drop Impact. Phys. Rev. Lett. 96:24501

${ }^{23}$ Keller J. B., Miksis M. J., (1983) Surface Tension Driven Flows. SIAM Journal on Applied Mathematics 43:268-277

${ }^{24}$ Zhang F. H., Thoroddsen S. T., (2008) Satellite generation during bubble coalescence. Phys. Fluids 20:022104

${ }^{25}$ Liger-Belair G., Villaume S., Cilindre C., Polidori G., Jeandet P., (2009) CO2 volume fluxes outgassing from champagne glasses in tasting conditions: flute versus coupe. J. Agric. Food Chem. 57:49394947

${ }^{26}$ Liger-Belair G., Conreux A., Villaume S., Cilindre C., (2013) Monitoring the losses of dissolved carbon dioxide from laseretched champagne glasses. Food Research International 54 54:516522

${ }^{27}$ Bosso A., Salmaso D., De Faveri E., Guaita M., Franceschi D., (2010) The use of carboxymethylcellulose for the tartaric stabilization of white wines, in comparison with other oenological additives . Vitis 49:95-99 